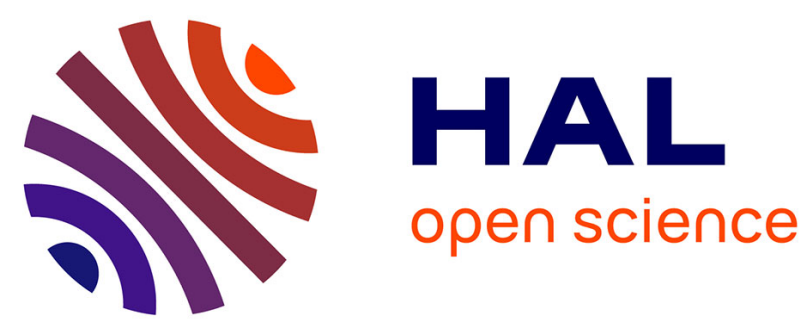

\title{
New Efficient Energy-Saving Techniques for Resource Allocation in Downlink OFDMA Transmission Systems
}

\author{
Joumana Farah, Elie Sfeir, Charbel Abdel Nour, Catherine Douillard
}

\section{To cite this version:}

Joumana Farah, Elie Sfeir, Charbel Abdel Nour, Catherine Douillard. New Efficient Energy-Saving Techniques for Resource Allocation in Downlink OFDMA Transmission Systems. ISCC 2017: The 22nd IEEE Symposium on Computers and Communications, Jul 2017, Crete, Greece. pp.1056 - 1062, 10.1109/ISCC.2017.8024665 . hal-01617338

\section{HAL Id: hal-01617338 \\ https://hal.science/hal-01617338}

Submitted on 10 Feb 2020

HAL is a multi-disciplinary open access archive for the deposit and dissemination of scientific research documents, whether they are published or not. The documents may come from teaching and research institutions in France or abroad, or from public or private research centers.
L'archive ouverte pluridisciplinaire $\mathbf{H A L}$, est destinée au dépôt et à la diffusion de documents scientifiques de niveau recherche, publiés ou non, émanant des établissements d'enseignement et de recherche français ou étrangers, des laboratoires publics ou privés. 


\title{
New Efficient Energy-Saving Techniques for Resource Allocation in Downlink OFDMA Transmission Systems
}

\author{
Joumana Farah ${ }^{(1)}$, Elie Sfeir ${ }^{(1)}$, Charbel Abdel Nour ${ }^{(2)}$, Catherine Douillard ${ }^{(2)}$ \\ (1) Department of Electricity and Electronics, Faculty of Engineering, \\ Lebanese University, Roumieh, Lebanon \\ ${ }^{(2)}$ IMT Atlantique, Department of Electronics, Lab-STICC - UMR 6285 \\ Technopôle Brest Iroise, CS 83818 - 29238 Brest Cedex, France
}

\begin{abstract}
This paper considers the problem of Base Station power minimization in downlink Orthogonal Frequency Division Multiple Access (OFDMA) systems. It aims at providing new solutions for the reduction of the total energy consumption. Such solutions rely on joint subcarrier and power allocation, under the constraints of requested per-user data rate. Practical results show that the proposed techniques allow a significant decrease of transmission power with an affordable complexity, compared to well-referenced methods from the literature.
\end{abstract}

Index Terms-OFDMA, power allocation, power reduction, subcarrier assignment, waterfilling.

\section{INTRODUCTION}

Nowadays, the proliferation of mobile devices and the constantly rising demands for high bandwidth-consuming services engage increasing amounts of gas emissions by the telecommunication sector. Also, Base stations (BS) constitute the main source of power consumption in mobile networks. The telecommunication industry contributes to more than 5\% of the worldwide $\mathrm{CO}_{2}$ emissions. From the economic standpoint, this energy consumption yields an important increase in the operational expenditure (OPEX) of mobile operators. Indeed, the energy cost of mobile operators constitutes more than $50 \%$ of their OPEX. These concerns have been pushing the technical community to find practical solutions that can decrease the amount of $\mathrm{CO}_{2}$ emissions of the telecommunication sector as well as energy costs supported by operators. Such environmental and financial issues can be partly tackled by proper resource (bandwidth and power) allocation strategies.

For this purpose, different strategies have been explored, in the past years, for the reduction of BS power, in the context of Orthogonal Frequency Division Multiple Access (OFDMA) systems. One of the first leading solutions was proposed in [1], where a quasi-optimal solution for the power minimization problem was developed: subcarriers are first assigned to users based on Lagrange optimization resolved by parameter relaxation. Transmission power and bit loading are then determined by a greedy approach. In [2], a lowcomplexity method is proposed to reduce the computational load of [1], by splitting the problem into two phases: resource allocation and subcarrier allocation. Several variants of the sub-optimal solution of [2] were proposed in [3-5]. They either aim at further reducing the complexity or slightly enhancing the performance of [2]. In previous works, we proposed waterfilling-based solutions for reducing the uplink power consumption or enhancing the downlink spectral efficiency of either orthogonal [6] or non-orthogonal [7-8] signaling systems.

In this paper, we aim at proposing novel solutions for joint subcarrier and power allocation that simultaneously seek the reduction of BS power and computational load. To this end, after a brief description of the system model in Section II, we develop a low-complexity recursive method for waterfilling optimization in Section III, in the context of power minimization. Then, we propose different resource allocation solutions that incorporate the recursive waterfilling method within the subcarrier assignment. Results and discussions follow in Section IV.

\section{RESOURCE ALLOCATION WITH POWER MINIMIZATION}

\section{A. System Description and Problem Formulation}

Consider a downlink system with $K$ mobile users randomly distributed over a single cell. Each user $k$ requests a transmission rate of $R_{k, \text { req }}$ [bps]. SISO (Single Input Single Output) transmissions are assumed throughout this study. System resources are shared by users using OFDMA, where the system bandwidth $B$ is equally divided into $S$ subcarriers. Let $P_{k, n}$ denote the power transmitted by the BS for user $k$ on subcarrier $n, R_{k, n}$ is the throughput achieved by user $k$ over $n$, $S_{\mathrm{k}}$ represents the set of subcarriers allocated to user $k, h_{k, n}$ is the frequency-domain channel coefficient between user $k$ and the BS over subcarrier $n$, and $N_{0}$ denotes the power spectral density of the additive white Gaussian noise (assumed to be constant over all subcarriers). The aim of this study is to 
develop allocation techniques that aim at providing each user its requested data rate with the minimum total amount of transmission power. The optimization problem can be formulated as follows:

$$
\begin{aligned}
& \left\{P_{k, n}, S_{k}\right\}^{*}=\underset{\left\{P_{k, n}, S_{k}\right\}}{\arg \min } \sum_{k} \sum_{n \in S_{k}} P_{k, n}, \text { subject to: } \\
& \sum_{n \in S_{k}} R_{k, n}=R_{k, r e q}, 1 \leq k \leq K, \\
& P_{k, n} \geq 0, \forall n \in S_{k}, 1 \leq k \leq K \\
& S_{i} \bigcap_{i \neq j} S_{j}=\varnothing, \\
& \bigcup_{k=1}^{K} S_{k} \subseteq\{1,2, \ldots, S\}
\end{aligned}
$$

The formulated problem is combinatorial and non-convex. In the sequel, we propose several allocation techniques that seek suboptimal solutions for the assignment problem.

\section{B. The Rate Craving Greedy (RCG) Solution}

This well-referenced sub-optimal technique was proposed in [2] as an efficient alternative to the high-complexity Lagrangian Relaxation quasi-optimal solution [1]. It consists of two stages: at the resource allocation stage, the average SNR (Signal to Noise Ratio) is computed for each user over all subcarriers. Then, based on the user-dependent requested rates and average SNRs, the necessary number of subcarriers is determined for each user. In the subsequent subcarrier allocation stage, the achievable rate is first computed, on each subcarrier, for each user. Rate computation requires the knowledge of the amount of power allocated to each subcarrier. Since users have individual rate constraints, Power Allocation (PA) is carried out, in our implementation of RCG, by applying a waterfilling procedure, separately for each user on all subcarriers, constrained by its requested rate. For this purpose, the gradual dichotomy-based waterfilling approach described in [6] is used. Then, each subcarrier is assigned to the user having the highest rate on it. After all subcarriers have been assigned, and as long as there are users assigned a number of subcarriers different than the one found at the first stage, subcarriers are transferred from users having too many subcarriers to the ones having less subcarriers than required. These transfers are performed in a way to minimize the rate difference between those subcarriers.

One of the disadvantages of the RCG algorithm is that the number of subcarriers per user is first determined in a separate stage, using the average SNR, which yields a suboptimum solution. In addition, it always uses the whole spectrum, including severely faded subcarriers. For this purpose, we propose several solutions that aim at jointly optimizing the distribution of subcarriers and minimizing the transmission power, so as to reduce the overall BS necessary power.

\section{PROPOSED ALLOCATION TECHNIQUES}

An efficient way to minimize the overall BS power is to allocate each subcarrier to the user that benefits the most from this allocation, i.e., for whom the power decrease is maximum under the target per-user rate constraint. However, the estimation of this power decrease for each candidate user, on the subcarrier $n$ considered for allocation, would require a separate waterfilling procedure for each candidate user, including the subcarrier $n$ and the user's formerly attributed subcarriers. Such strategy could lead to a prohibitive amount of calculations.

For this purpose, we will start in Section IIIA by developing a low-complexity recursive method for estimating the power decrease, in order to incorporate it into our allocation methods.

\section{A. Recursive waterline and power decrease estimation}

Let $P_{k, t o t}^{(1)}$ and $P_{k, t o t}^{(2)}$ denote the total amount of necessary power for a user $k$, before and after the assignment of a subcarrier to $k$. In the proposed solutions, the criteria used for subcarrier assignment are based on the amount of power decrease, $\Delta P_{k, n}=P_{k, t o t}^{(2)}-P_{k, t o t}^{(1)}$, that this assignment may induce for user $k$. In order to determine $\Delta P_{k, n}$, PA needs to be applied for each candidate user (for a fixed subcarrier), or for each candidate subcarrier (for a fixed user), before determining the optimal assignment. Such power estimations would yield a prohibitive computational load. For this purpose, we propose in this paper a recursive low-complexity method for estimating the new waterline level after each subcarrier assignment, as well as the corresponding $\Delta P_{k, n}$.

For any user $k$, the PA can be formulated as the solution of the optimization problem:

$\operatorname{Min}_{\left\{P_{k, n}\right\}} \sum_{n \in S_{k}} P_{k, n}$, such that:

$$
\sum_{n \in S_{k}} \frac{B}{S} \log _{2}\left(1+\frac{P_{k, n} h_{k, n}^{2}}{N_{0} B / S}\right)=R_{k, r e q}
$$

Using the standard Lagrangian optimization, this leads to the well-known waterfilling solution where $P_{k, n}$ can be written as:

$P_{k, n}=\max \left\{\frac{B \lambda_{k}\left(N_{k}\right)}{S \ln 2}-\frac{N_{0} B / S}{h_{k, n}^{2}}, 0\right\}$, where $\lambda_{k}\left(N_{k}\right)$ is the

Lagrange multiplier for user $k$ when the number of its allocated subcarriers is $N_{k}=\operatorname{Card}\left(S_{k}\right)$. The corresponding waterline is: $w_{k}\left(N_{k}\right)=B \lambda_{k}\left(N_{k}\right) / S \ln 2$.

By replacing the expression of $P_{k, n}$ into (1), we obtain:

$w_{k}\left(N_{k}\right)=(B / S \ln 2) 2^{\frac{1}{N_{k}}\left[\frac{S}{B} R_{k, \text { req }}-\sum_{n \in S_{k}} \log _{2}\left(\frac{h_{k, n}^{2}}{N_{0} \ln 2}\right)\right]}$.

When a subcarrier $n_{a}$ is added to $k$, the waterline becomes:

$w_{k}\left(N_{k}+1\right)=(B / S \ln 2) 2^{\frac{1}{N_{k}+1}\left[\frac{S}{B} R_{k, \text { req }}-\sum_{n \in S_{k} \cup\left\{n_{a}\right\}} \log _{2}\left(\frac{h_{k, n}^{2}}{N_{0} \ln 2}\right)\right]}$.

By writing $R_{k, \text { req }}$ in terms of $w_{k}\left(N_{k}\right)$ and replacing it into the expression of $w_{k}\left(N_{k}+1\right)$, we obtain, after some manipulations: 


$$
w_{k}\left(N_{k}+1\right)=\frac{\left(w_{k}\left(N_{k}\right)\right)^{N_{k} / N_{k}+1}}{\left(\frac{h_{k, n_{a}}^{2}}{N_{0} B / S}\right)^{1 / N_{k}+1}} .
$$

After the assignment of the first subcarrier $n_{l}$ to user $k$, the initial waterline level can be found as the sum of the inverse channel gain with the necessary power to achieve $R_{k, \text { req }}$ on subcarrier $n_{l}$ alone. It is therefore:

$$
w_{k}(1)=\frac{N_{0} B / S}{h_{k, n_{1}}^{2}}+\left(2^{\frac{R_{k, \text { req }}}{B / S}}-1\right) \frac{N_{0} B / S}{h_{k, n_{1}}^{2}}=\frac{N_{0} B / S}{h_{k, n_{1}}^{2}} 2^{\frac{R_{k, \text { req }}}{B / S}} \text {. }
$$

Using (2), it can be verified that adding subcarrier $n_{a}$ decreases the waterline, i.e. $w_{k}\left(N_{k}+1\right)<w_{k}\left(N_{k}\right)$, only if its channel gain verifies the condition: $h_{k, n_{a}}^{2}>\frac{N_{0} B / S}{w_{k}\left(N_{k}\right)}$.

As for $\Delta P_{k, n}$, it can be obtained by writing $P_{k, t o t}^{(1)}$ and $P_{k, t o t}^{(2)} a s$ :

$$
\begin{aligned}
& P_{k, t o t}^{(1)}=N_{k} w_{k}\left(N_{k}\right)-\sum_{n \in S_{k}} \frac{N_{0} B / S}{h_{k, n}^{2}}, \\
& P_{k, t o t}^{(2)}=\left(N_{k}+1\right) w_{k}\left(N_{k}+1\right)-\sum_{n \in S_{k} \cup\left\{n_{a}\right\}} \frac{N_{0} B / S}{h_{k, n}^{2}}, \text { leading to: } \\
& \Delta P_{k, n}=\left(N_{k}+1\right) w_{k}\left(N_{k}+1\right)-N_{k} w_{k}\left(N_{k}\right)-\frac{N_{0} B / S}{h_{k, n_{a}}^{2}} .
\end{aligned}
$$

\section{B. The Random SubCarrier-Minimum Decrease of Power (RSC-MinDP) Solution}

In this first allocation method, subcarriers are considered one by one, in a random order, so as to select the best user to be assigned for each one (Fig. 1). The best user $k^{*}$ allocated to a subcarrier $n$, from the set of available subcarriers $S_{p}$, is selected as $k^{*}=\underset{k}{\arg \min } \Delta P_{k, n}$, where $\Delta P_{k, n}<0$. However, only users whose channel gains verify (3) are considered as candidate users over $n$. Otherwise, adding this subcarrier to the candidate user would increase its power.

On the other hand, it may happen that the allocation of a subcarrier to user $k^{*}$ only decreases its necessary power by a negligible amount. For this purpose, the following condition is tested before subcarrier assignment: $\Delta P_{k^{*}, n}<-\rho$, where the value of the positive threshold $\rho$ is chosen in such a way to strike a balance between the system power efficiency and spectral efficiency, since unallocated subcarriers may be used by other operators or cognitive systems.

Furthermore, each time a new subcarrier is added to a user $k^{*}$, we test if $k^{*}$ has a zero amount of power on one or more of its previously assigned subcarriers (because of the waterline decrease). If this is the case, such subcarriers are returned to the set of available subcarriers in order to be subsequently allocated by the algorithm. However, such cases rarely occur.

The algorithm ends when no more subcarriers can be assigned, because they do not lead to a significant decrease in power to any user, or when no more subcarriers are available. Note that the random order in assigning the subcarriers aims at benefiting from frequency diversity so as to avoid the allocation of neighboring (subsequent) subcarriers to the same user.
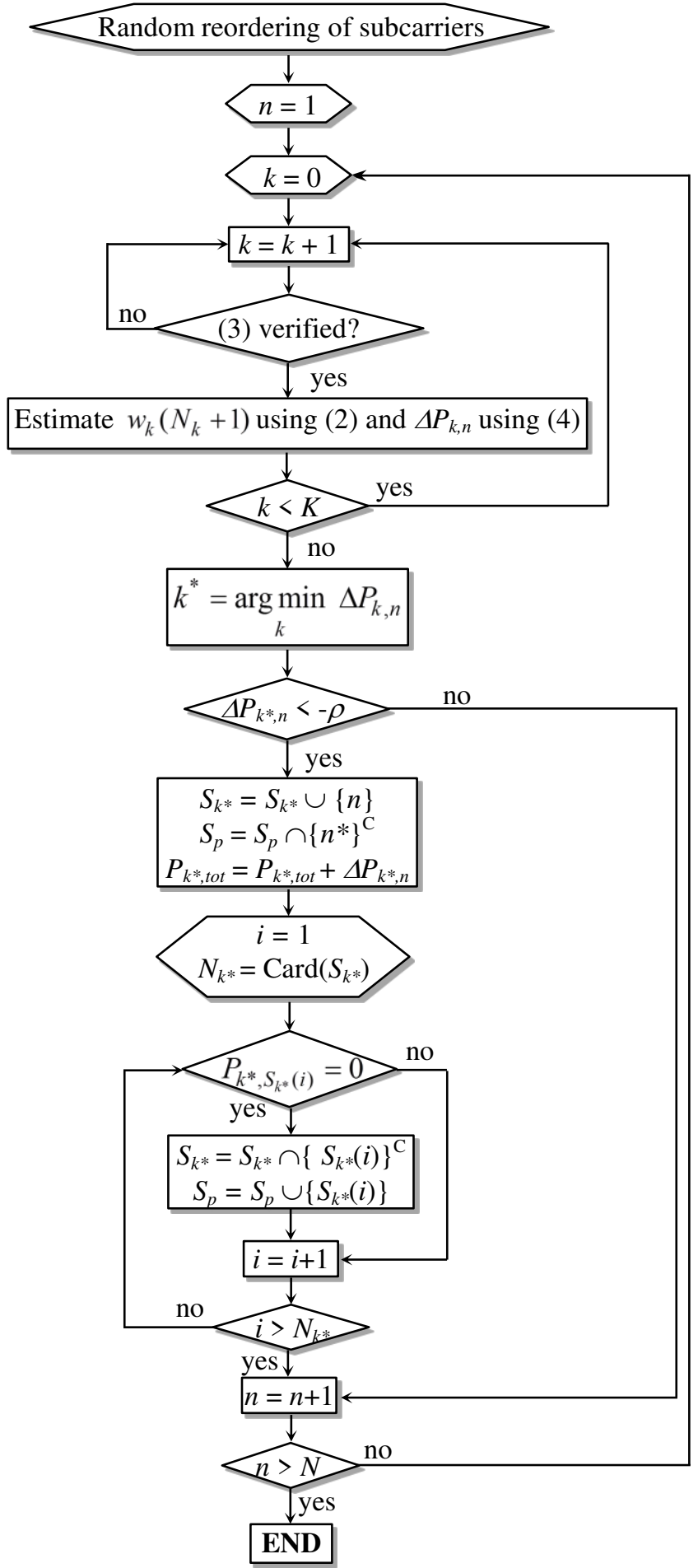

Fig. 1. RSC-MinDP allocation technique.

C. The Random SubCarrier - Initialization with MaxH Minimum Decrease of Power (RSC-IMaxH-MinDP) Solution

In this method, we also go through the subcarriers in a 
random order, to select the corresponding assigned user. However, an initialization phase, called "Worst-Best-H" assignment, is added at the beginning of the procedure, as was done in the allocation techniques of [7-8]. The aim of enhancing the initialization is to avoid depriving cell-edge users of their best subcarriers (essential in decreasing their power) in favor of cell-center users. This effect was observed with the RSC-MinDP technique. The initialization proceeds as follows: for each user $k$, the subcarrier with the highest channel gain $h_{k, \text { best }}$ is first determined. The user with the highest priority is the one having the lowest $h_{k, \text { best }}$. This user is then assigned its best subcarrier. Then, this process is applied to the user with the next priority, and so on, until each user has one allocated subcarrier. At this point, the initialization process is terminated, and subcarriers are now assigned using the same steps as in the previous RSC-MinDP method.

\section{The Random SubCarrier - Initialization with MaxH - Maximum (-DeltaP/P $\left.{ }^{\alpha}\right)\left(\right.$ RSC-IMaxH-Max $\left.\left(-D P / P^{\alpha}\right)\right)$ Solution}

A drawback of the RSC-IMaxH-MinDP solution is that cell-edge users, because of their high power needs, often lead to the lowest values of $\Delta P_{k, n}$ among users and are attributed a large number of subcarriers. Therefore, they may prevent the BS from efficiently decreasing the power of cell-center users, leading to a high overall BS power. In order to alleviate this problem, the decision metric in this method consists in maximizing $-\Delta P_{k, n} / P_{k, \text { tot }}^{\alpha}$ instead of minimizing $\Delta P_{k, n}$, where $P_{k, t o t}$ is the new power level reached if subcarrier $n$ is assigned to user $k$, and $\alpha$ a positive exponent. This allows the user having the lowest new power level to be favored over subcarrier $n$. In fact, a low (resp. high) value of $\alpha$ tends to favor cell-edge (resp. cell-center) users with respect to others.

\section{E. The Maximum User Power - Minimum Decrease of Power (MaxP-MinDP) Solution}

In this fourth proposed solution (Fig. 2), instead of going through the subcarriers in a random manner, at each allocation stage, a user $k^{*}$ is first chosen according to a priority assignment. For the first $K$ assigned subcarriers, priority is based on the Worst-Best-H principle, as in the two previous methods. Once each user has one assigned subcarrier, priority is now based on the actual necessary transmission power for each user: user $k^{*}$ with the highest required power is selected to be attributed a new subcarrier from set $S_{p} . k^{*}$ is assigned the subcarrier $n^{*}$ that leads to the lowest decrease of power. Only subcarriers verifying (3) are considered as candidate subcarriers for $k^{*}$. Also, subcarrier $n^{*}$ is assigned to $k^{*}$ only if it verifies $\Delta P_{k^{*}, n^{*}}<-\rho$. Note that, since user $k^{*}$ is fixed, minimizing $\Delta P_{k^{*}, n^{*}}$ is equivalent to minimizing the new user's allocated power level after subcarrier allocation.

Furthermore, if $\Delta P_{k^{*}, n^{*}}>-\rho, k^{*}$ is removed from the set of users $U_{p}$ whose power level can still be decreased, i.e., $k^{*}$ will no longer be considered for further subcarrier assignments. Subcarriers with zero powers are checked and freed before the user is removed from $U_{p}$. The algorithm stops when no more subcarriers are available or when the set $U_{p}$ is empty.

In order to decrease complexity, and since for all considered subcarriers, $N_{k^{*}} w_{k^{*}}\left(N_{k^{*}}\right)$ is the same when calculating $\Delta P_{k^{*}, n^{*}}$, only the first and third terms in (4) are considered for determining the most favorable subcarrier for user $k^{*}$.

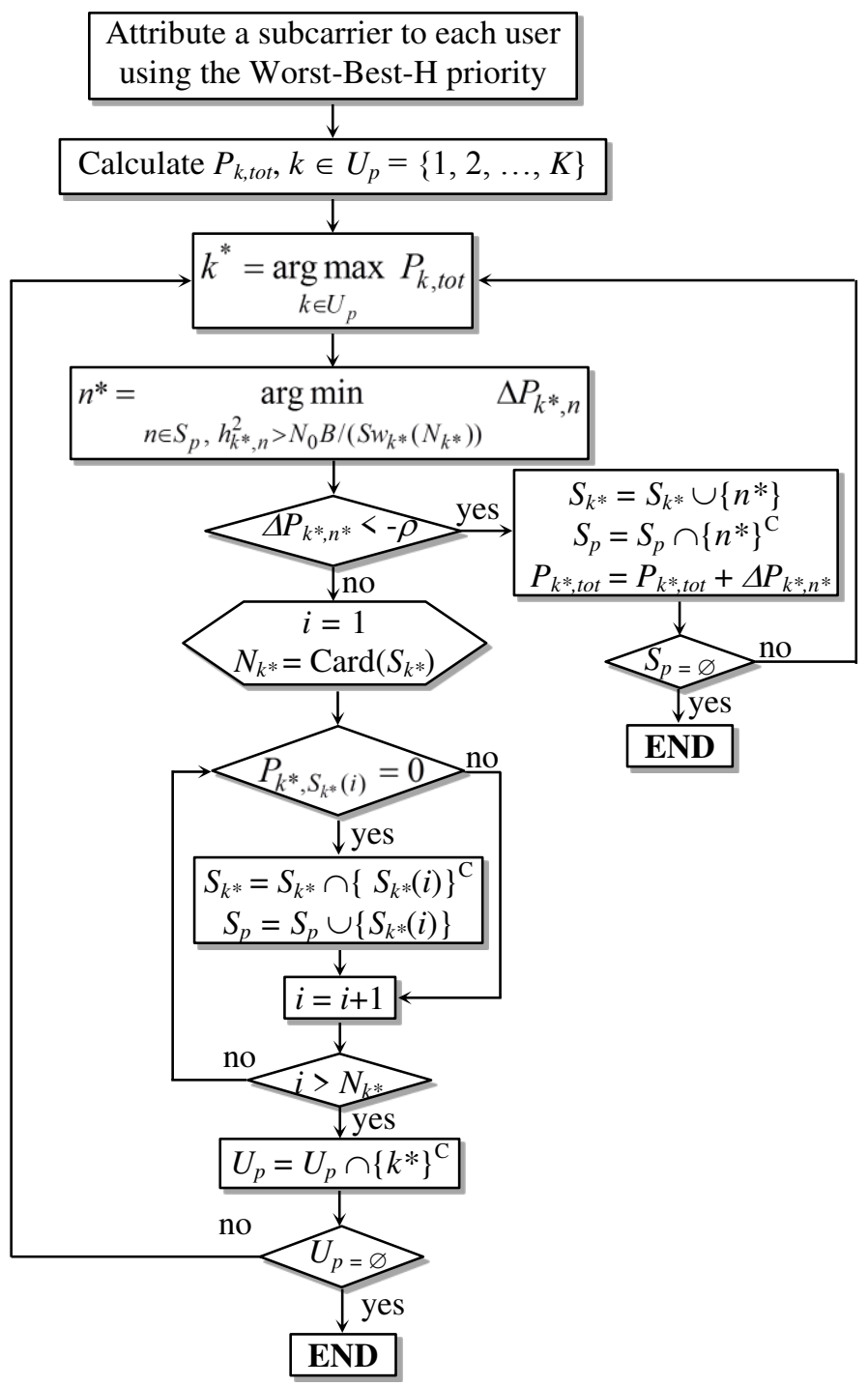

Fig. 2. MaxP-MinDP allocation technique.

\section{PRACTICAL RESULTS}

In this section, we analyze the performance of the proposed techniques under several practical scenarios. We also compare our results with those obtained using the RCG technique that serves as a benchmark in a plethora of previous works in the field [2-5].

The performance of the different methods are assessed by intensive simulations, in the following conditions: the cell radius is $500 \mathrm{~m}$ and the system bandwidth $B$ is $10 \mathrm{MHz} . K$ varies between 10 and 28, $S$ between 16 and 512, and the requested user rates $R_{k, \text { req }}$ between 5 and $15 \mathrm{Mbps}$. The 
transmission medium is modeled by a frequency-selective Rayleigh fading channel with a rms of 500 ns. Distancedependent path loss with a decay factor of 3.76 is considered. Perfect knowledge of the user channel gain by the BS is assumed in this study, considering regular CSI (Channel State Information) transmissions from the MS (Mobile Stations) to the BS. $N_{0}$ is $4.10^{-18} \mathrm{~mW} / \mathrm{Hz}$.

The first set of practical tests aims at determining the optimal value of the threshold $\rho$ used in our allocation techniques. For this purpose, Fig. 3 shows the total necessary power as a function of the parameter $\rho$, for different values of the number of users, using the MaxP-MinDP technique with a number of subcarriers $S=128$ and a per-user target rate $R_{k, \text { req }}=5$ Mbps. Fig. 4 shows the corresponding total number of occupied subcarriers.

As it can be seen from the simulation results, increasing the threshold $\rho$ increases the necessary total power, since the algorithms tend to allocate subcarriers less often to users. This is also verified in the results of Fig. 4, which shows that, starting from a value of $\rho$ of $0.001 \mathrm{~W}$, the total number of used subcarriers decreases quickly.

Also, it should be noted that increasing the value of the threshold decreases the complexity, since users tend more often to leave the set of active users $U_{p}$. Based on these observations, a value of $\rho=0.01 \mathrm{~W}$ is chosen in the following simulations, since it ensures an acceptable tradeoff between the necessary power and the occupied spectrum, for the different values of $K$. At this value of $\rho$, for 10 users with a requested rate of $5 \mathrm{Mbps}$, around $66 \%$ of the subcarriers are used by our proposed allocation techniques, whereas RCG uses the whole spectrum.

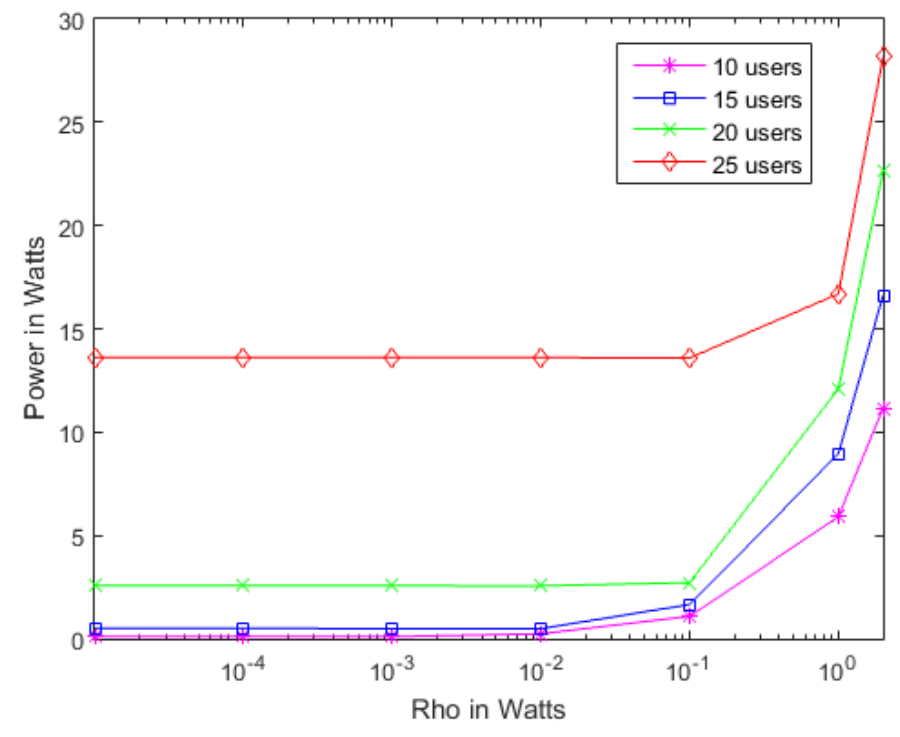

Fig. 3. Total power as a function of the threshold $\rho$, for $S=128$ and $R_{k, \text { req }}=5 \mathrm{Mbps}$.

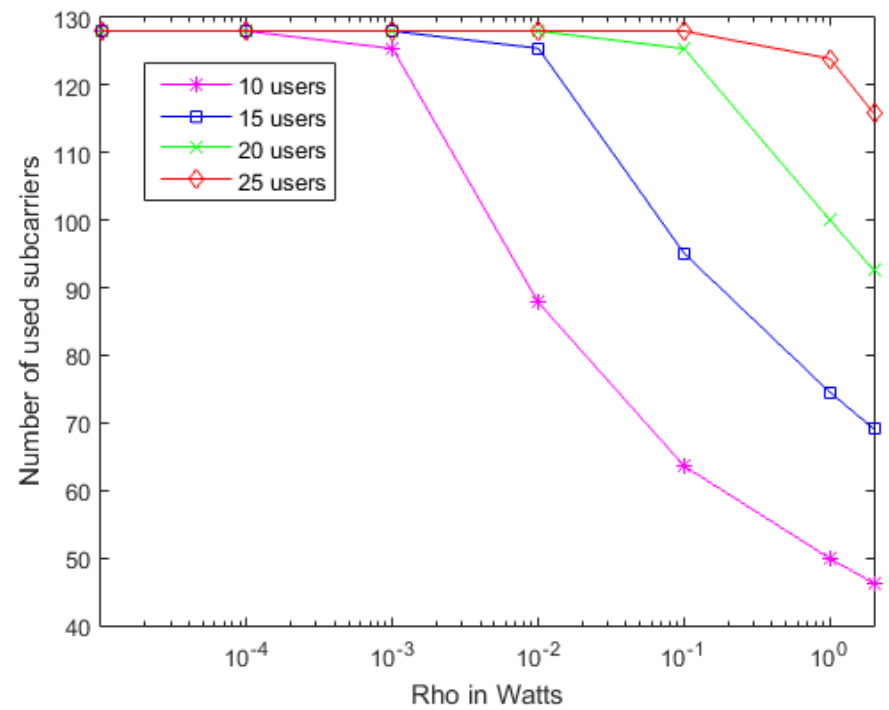

Fig. 4. Total number of used subcarriers as a function of the threshold $\rho$, for $S=128$ and $R_{k, \text { req }}=5 \mathrm{Mbps}$.

Next, we move to determining the optimal value of parameter $\alpha$ used in the RSC-IMaxH-Max $\left(-\mathrm{DP} / \mathrm{P}^{\alpha}\right)$ technique. For this purpose, Fig. 5 shows the total power as a function of $\alpha$, for a number of subcarriers $S=128$, and different combinations of the number of users $K$ and the user target rate $R_{k, r e q}$.

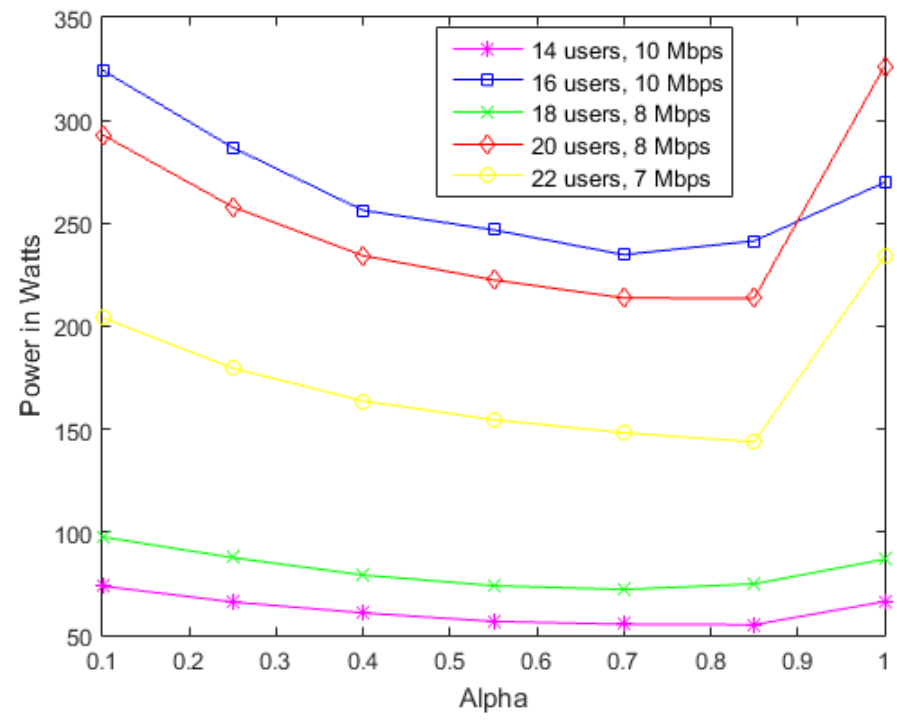

Fig. 5. Total power as a function of parameter $\alpha$, for $S=128$.

In fact, a low value of $\alpha$ gives more strength to the $\Delta P$ term in the decision metric of the RSC-IMaxH-Max (-DP/ $\left.\mathrm{P}^{\alpha}\right)$ technique, and therefore tends to favor cell-edge users with respect to cell-center users. On the contrary, a high value of alpha tends to favor cell-center users. This explains the presence of a minimum value that constitutes the best tradeoff between favoring cell-edge and cell-center users, and that corresponds to the lowest level of the necessary power. This 
level corresponds to an optimal value of $\alpha$ generally around 0.8 that will be adopted for this algorithm, in the subsequent tests.

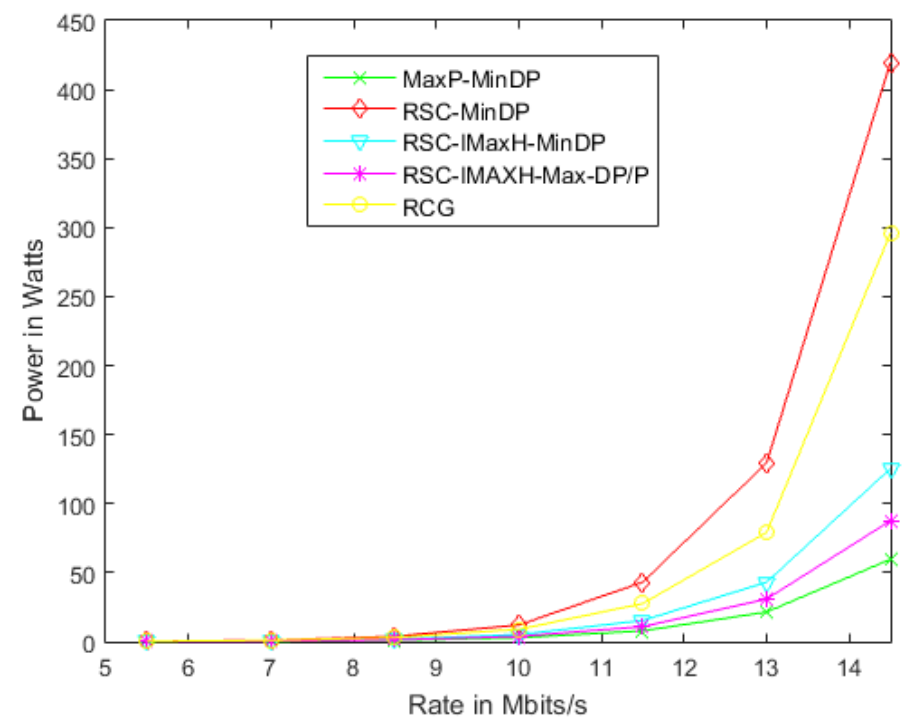

Fig. 6. Total power as a function of $R_{k, \text { req }}$, for $K=10$ and $S=128$.

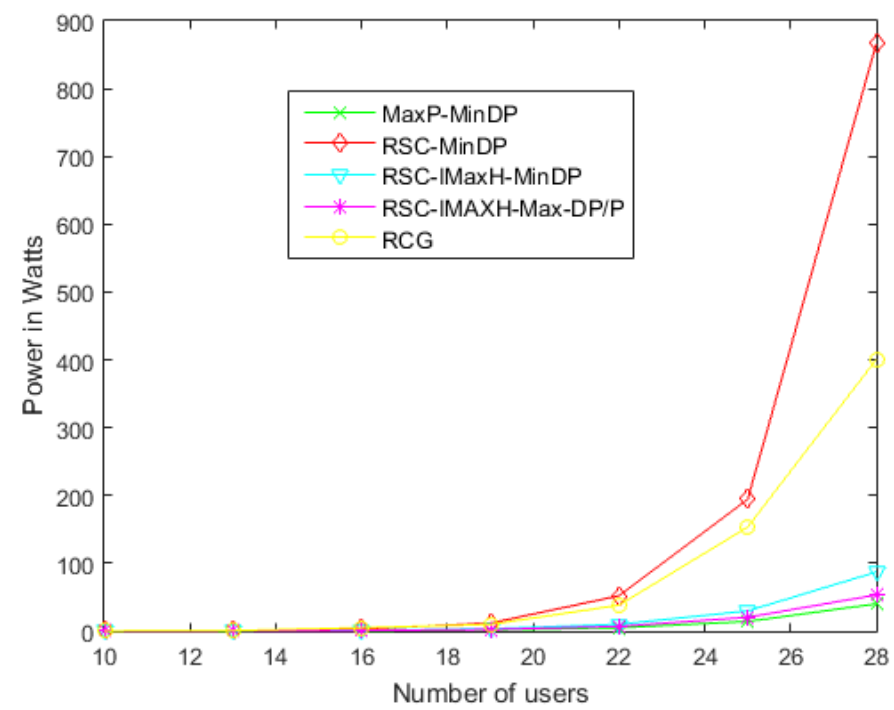

Fig. 7. Total power as a function of $K$, for $S=128$ and $R_{k, \text { req }}=5$ Mbps.

Fig. 6 shows the total BS transmission power in terms of $R_{k, \text { req }}$, for a number of users $K=10$ and a number of subcarriers $S=128$. It is clear that, except for RSC-MinDP, all proposed methods greatly outperform RCG, with a gain that largely increases as $R_{k, \text { req }}$ increases. RSC-MinDP suffers from the absence of a proper initialization stage, and from the fact that many subcarriers are taken by cell-edge users. Compared to RCG, MaxP-MinDP allows a reduction of the total power by an amount that can reach $240 \mathrm{~W}$ at a target rate of 14.5 Mbps.
Fig. 7 represents the total power as a function of $K$, for 128 subcarriers and a per-user target rate of $5 \mathrm{Mbps}$. It shows that MaxP-MinDP allows 28 users to reach a target rate of $5 \mathrm{Mbps}$ with a BS power almost equal to the $40 \mathrm{~W}$ preconized by the LTE (Long Term Evolution) standard [9], whereas RSCIMaxH-Max $\left(-\mathrm{DP} / \mathrm{P}^{\alpha}\right)$ and $\mathrm{RCG}$ respectively require 54 and $400 \mathrm{~W}$.

Fig. 8 depicts the total power in terms of the total number of subcarriers, for the case of 10 users and a per-user target rate of $10 \mathrm{Mbps}$. It shows that, at a low number of subcarriers $(S=16)$, RCG necessitates a BS power higher than $100 \mathrm{~W}$, to serve 10 users at $10 \mathrm{Mbps}$, whereas all proposed methods are under $40 \mathrm{~W}$. Starting from 32 subcarriers, the necessary power by RSC-IMaxH-Max(-DP/ $\left.\mathrm{P}^{\alpha}\right)$ and MaxP-MinDP is respectively 4.5 and $3.2 \mathrm{~W}$.

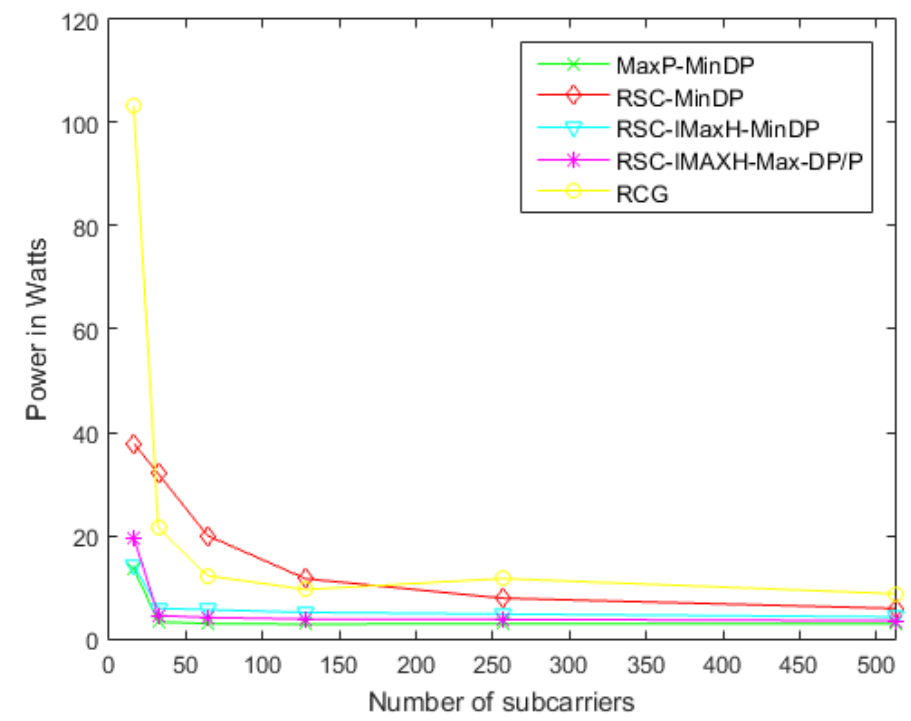

Fig. 8. Total power as a function of $S$, for $K=10$ and $R_{k, \text { req }}=10$ Mbps.

Regarding the algorithmic complexity, the four proposed techniques have a complexity dominated by $\mathrm{O}(K S)$, since the waterfilling procedure is replaced by recursive estimations. As for RCG, its complexity is dominated by $\mathrm{O}(K S \log (S))$, since rate estimation in the second step is performed by a waterfilling procedure for each user on all subcarriers. Moreover, we measured the average execution time of one complete allocation cycle on a Desktop Intel Core2 Quad CPU $2.83 \mathrm{GHz}$. For $K=10, S=32$, and $R_{k, r e q}=14.5 \mathrm{Mbps}$, MaxPMinDP, RSC-MinDP, RSC-IMaxH-MinDP, RSC-IMaxH$\operatorname{Max}\left(-\mathrm{DP} / \mathrm{P}^{\alpha}\right)$, and $\mathrm{RCG}$ take respectively an average of 9.03 , 10.74, 15.92, 17.16, and 32.01 milliseconds.

\section{CONCLUSION}

In this paper, four resource allocation methods were proposed for the reduction of BS power in a downlink OFDMA system, under the constraints of user-dependent requested rate. Simulation results show substantial gains with 
three methods when compared to the well-known RCG technique, with a lower complexity. Also, an interesting result is that it is always better to go through users, prioritized by their necessary power level, than going through subcarriers in random order. We are currently undergoing research to apply our study in the contexts of Non-Orthogonal Multiple Access (NOMA) systems and Multiple-Input Multiple-Output (MIMO) systems. The influence of imperfect channel estimation will also be considered.

\section{ACKNOWLEDGEMENT}

This work has been funded with support from the Lebanese University. Part of this work has been performed in the framework of the Horizon 2020 project FANTASTIC-5G (ICT-671660), which is partly funded by the European Union.

\section{REFERENCES}

[1] C. Y. Wong, R. S. Cheng, K. Ben Letaief, and R. D. Murch, "Multiuser OFDM with Adaptive Subcarrier, Bit, and Power Allocation", IEEE Journal on Sel. Areas in Comm., vol. 17, no. 10, Oct. 1999, p. 1747-58.

[2] D. Kivanc, G. Li, and H. Liu, "Computationally Efficient Bandwidth Allocation and Power Control for OFDMA", IEEE Trans. Wireless Comm., Vol. 2, No. 6, Nov. 2003, p. 1150-58.

[3] Y.-B. Lin, T.-H. Chiu, and Y. T. Su, "Optimal and NearOptimal Resource Allocation Algorithms for OFDMA Networks", IEEE Trans. Wireless Comm., Vol. 8, No. 8, August 2009, pp. 4066-4077.

[4] C. Xiong, G. Y. Li, S. Zhang, Yan C., and S. Xu, "Energy- and Spectral-Efficiency Tradeoff in Downlink OFDMA Networks", IEEE Trans. Wireless Comm., Vol. 10, No. 11, Nov. 2011, pp. 3874-3886.

[5] H. Holtkamp, G. Auer, S. Bazzi, and H. Haas, "Minimizing Base Station Power Consumption", IEEE Journal Sel. Areas Comm., Vol. 32, No. 2, February 2014, pp. 297-306.

[6] J. Farah and F. Marx, "Combining strategies for the optimization of resource allocation in a wireless multiuser OFDM system", AEU Inter. Journal of Elect. and Comm., Vol. 61, No. 10, pp. 665 - 677, 2007.

[7] M.R. Hojeij, J. Farah, C. Abdel Nour, and C. Douillard, "New Optimal and Suboptimal Resource Allocation Techniques for Downlink Non-Orthogonal Multiple Access", Wireless Pers. Commun., 2015, pp. 1-31.

[8] M.R. Hojeij, J. Farah, C. Abdel Nour, and C. Douillard, "Resource Allocation in Downlink Non-Orthogonal Multiple Access (NOMA) for Future Radio Access", in proc. 81st IEEE VTC, 2015.

[9] 3GPP, TR25-814 (V7.1.0), Physical Layer Aspects for Evolved UTRA (2006). 